\title{
Insomnia Symptoms in Chinese Patients with Chronic Schizophrenia: Prevalence, Clinical Correlates and Relationship with Inflammation
}

\section{Lei Xia}

Chaohu hospital of Anhui Medical University

\section{Yi Zhong}

Chaohu hospital of Anhui Medical University

Zhiwei Liu

Chaohu Hospital of Anhui Medical University

\section{Yulong Zhang}

Chaohu Hospital of Anhui Medical University

\section{Wenzheng Li}

Hefei Fourth People's Hospital

\section{Yun Wang}

Chaohu Hospital of Anhui Medical University

\section{Degang Tao}

\section{Maanshan Fourth People's Hospital}

\section{Kai Zhang}

Chaohu Hospital of Anhui Medical University

Huanzhong Liu ( $\nabla$ huanzhongliu@ahmu.edu.cn )

Chaohu Hospital of Anhui Medical University https://orcid.org/0000-0003-3095-5249

\section{Xiang Yang Zhang}

Chinese Academy of Sciences

\section{Primary research}

Keywords: Schizophrenia, Insomnia, Risk factors, Inflammation, China

Posted Date: July 16th, 2020

DOI: https://doi.org/10.21203/rs.3.rs-43724/v1

License: (a) (i) This work is licensed under a Creative Commons Attribution 4.0 International License.

Read Full License 


\section{Abstract}

Background: Sleep disturbances are common in patients with schizophrenia, with serious consequences. The purpose of this study was to investigate the prevalence and clinical correlates of insomnia symptoms, and to explore the relationship between insomnia and inflammatory markers in Chinese patients with chronic schizophrenia.

Methods: A total of 328 inpatients with chronic schizophrenia were recruited. Insomnia Severity Index (ISI), Calgary Depression Scale for Schizophrenia (CDSS), and Positive and Negative Syndrome Scale (PANSS) were used to assess the severity of insomnia, depression, and psychotic symptoms. The plasma levels of several inflammatory markers (CRP, IL-6, and TNF-a) were measured.

Results: The prevalence of insomnia symptoms in patients with schizophrenia was $38.4 \%$. Depressive symptoms were significantly associated with insomnia symptoms $(\mathrm{OR}=1.23,95 \% \mathrm{Cl}$ : 1.13-1.33, $P<$ $0.001)$. Higher CDSS score (beta $=0.55, \mathrm{t}=8.21, P<0.001)$ and older age (beta $=0.06, \mathrm{t}=3.59, P<0.001)$ were significantly associated with higher ISI score, while taking a single SGA (beta $=-0.85, \mathrm{t}=-1.99, P<$ 0.05 ) was independently associated with lower ISI score. There was no significant association between any inflammatory markers and insomnia or ISI score.

Conclusions: Our results demonstrate that the prevalence of insomnia symptoms is high in Chinese inpatients with chronic schizophrenia. Some demographic and clinical variables, such as depressive symptoms and older age, are risk factors, while others are beneficial factor, such as taking atypical antipsychotic drug for insomnia in schizophrenia patients. No association has been found between insomnia symptoms and inflammation.

\section{Background}

Insomnia is a common sleep disorder characterized by dissatisfaction with the quality or quantity of sleep. It usually manifests as having difficulty falling asleep, waking up frequently, and waking up too early $[1,2]$. According to different definitions, the prevalence of insomnia varies considerably in different epidemiological studies. In the general population, $6 \%-9.5 \%$ of people meet the insomnia criteria of the 4th edition of Diagnostic and Statistical Manual of Mental Disorder (DSM-IV), while approximately 30\% report having insomnia symptoms without strict criteria assessment [2-4]. Individuals with insomnia are more likely to be absent from work, have more accidents in their lives, and take up more medical and health resources than people with good sleep quality [5].

Sleep disturbances usually occur in patients with schizophrenia [6], which may have existed before the onset of the disease and are highly associated with acute exacerbation of psychotic symptoms [7]. A oneyear follow-up study [8] of adolescents at high risk for psychiatric disorders found that circadian rhythm disorders predicted the severity of psychotic symptoms and psychosocial damage. Furthermore, in patients with schizophrenia, insomnia may lead to a significant reduction in quality of life [9] and a more 
than 10-fold increase in the risk of suicide [10]. There is no doubt that insomnia is very harmful to this group of population and is worthy of attention.

Difficulties in falling asleep and maintaining sleep are the two most common manifestations of insomnia in schizophrenia, which may be due to overactivity of the dopaminergic system and dysfunction of the GABA system [11]. Regarding the effects of antipsychotic administration on sleep architecture, secondgeneration antipsychotics (SGAs) tend to ameliorate insomnia in patients with schizophrenia [12]. However, antipsychotics act by blocking $D_{2}$ receptors, which may exacerbate restless leg syndrome and periodic limb movement disorder, ultimately worsening sleep quality [7].

Sleep plays a homeostatic role in the regulation of inflammatory biology dynamics. Sleep disturbances, including insomnia, can activate the innate immune system at multiple levels and promote the production of proinflammatory cytokines [13]. There has been many studies on the relationship between sleep disturbances (including poor sleep quality, and insomnia complaints) and inflammatory markers [14], but few studies have focused on specific populations [15]. A recent study was considered to be the first report to show an association between sleep quality and inflammatory response in schizophrenia by measuring neutrophil-lymphocyte and platelet-lymphocyte ratios, but not levels of inflammatory cytokines [16]. Another more recent study found that sleep quality was related to CRP and IL-6 levels in outpatients with schizophrenia, but did not use any validated sleep questionnaires [17].

To date, only a few studies $[9,18,19]$ have investigated insomnia in Chinese outpatients or community patients with schizophrenia, with inconsistent insomnia rates, ranging from $19.3-36.0 \%$. There is no data on insomnia in Chinese hospitalized patients with chronic schizophrenia. Also, there is no report on the relationship between insomnia and inflammatory cytokines in these patients. Therefore, the main purposes of this study were 1) to examine the prevalence, socio-demographic and clinical correlates of insomnia in patients with chronic schizophrenia, and 2) to explore the relationship between insomnia and inflammatory markers in these patients.

\section{Methods}

\section{Study Design and Participants}

This cross-sectional study was part of a survey of physical diseases and psychological status in inpatients with schizophrenia (Trial registration: www.chictr.org.cn, ChiCTR1800017044). The survey was conducted in three hospitals: Chaohu Hospital of Anhui Medical University, Hefei Fourth People's Hospital, and Maanshan Fourth People's Hospital, which have a total of 1500 psychiatric beds, and serve more than 5 million people in Anhui Province, China. Patients were recruited consecutively from May 2018 to December 2018, provided that they met the following criteria: 1) aged $\geq 18$ years; 2) met diagnosis of schizophrenia based on the 10th revision of the International Statistical Classification of Diseases and Related Health Problems (ICD-10) [20];3) with $\geq 5$ years of disease course; and 4) no pregnancy or 
breastfeeding. People were excluded if they had 1) serious nervous system diseases, mental retardation, or dementia; or 2) immune diseases, or taking anti-inflammatory drugs.

\section{Data collection}

\section{Socio-demographic characteristics}

Each participant completed a predesigned questionnaire to collect socio-demographic and clinical variables such as age, sex, marital status, education, smoking behavior, antipsychotic (APs) and benzodiazepine (BZDs) use, and detailed hospitalization information. The data of all inpatients were extracted from electronic medical records. The chlorpromazine equivalent (CPZeq) of the APs dose was calculated in the defined daily doses (DDDs) method [21]. In this study, patients with hypertension and diabetes, were considered to have chronic somatic diseases (CSDs) through hospital diagnosis and corresponding medications (antihypertensive, hypoglycemic drugs, etc.).

\section{Insomnia assessment}

Under the guidance of the researchers, participants completed some clinical questionnaires through selfassessment, and conducted clinical interviews when necessary. Following the methods of previous studies [22, 23], three sleep items (4, 5, and 6) of the Hamilton Depression Rating Scale (HAM-D) were used to assess sleep disturbances in the previous 2 weeks, and three types of insomnia were identified: early, middle and late insomnia. These items were scored on a 3-point scale, from $0=$ no difficulty to $2=$ severe difficulty. If a patient was rated as $\geq 1$ on any of the three items, he or she was defined as "having insomnia symptom".

Insomnia Severity Index (ISI) [24] is a 7-item self-rating questionnaire designed to assess the severity of insomnia symptoms in different populations, including patients with schizophrenia. Its seven items are scored on a 4-point scale from 0 to 4, and the higher the total score, the more severe the symptoms of insomnia. Because the optimal cut-off score for ISI is still uncertain [25], in this study, the ISI total score was only used to assess the severity of insomnia symptoms.

Furthermore, the seven items of ISI are divided into three components: Factor 1 is labeled as "Impact" (interference, noticeability, and distress), Factor 2 is labeled as "Severity" (sleep onset, sleep maintenance, and early morning awakening), and Factor 3 is labeled as "Satisfaction" (sleep onset, satisfaction, and distress). The three-factor structure was supported by confirmatory factor analysis in previous studies [26].

\section{Assessments of depressive and psychotic symptoms}

Calgary Depression Scale for Schizophrenia (CDSS) is a 9-item scale, which has been proved to be a reliable and effective tool for the assessment of depressive symptoms in Chinese patients with schizophrenia [27]. The total score of the CDSS ranges from 0 to 27 . As in the previous study, the 7 th item requiring early awakening, was removed from the data analysis [28]. 
The Positive and Negative Syndrome Scale (PANSS) [29] was used to measure positive symptoms (items P1-7), negative symptoms (items N1-7), and general psychopathology symptoms (items G1-16) in schizophrenia patients with schizophrenia. The subscales were abbreviated as "PANSS-P", "PANSS-N" and "PANSS-G", respectively. Four psychiatrists participated in scoring PANSS, and received a training course in the use of PANSS before the the study began. Their inter-rater correlation coefficient was 0.9 for the PANSS total score.

\section{Body Mass Index (BMI), fasting blood glucose (FBG), and inflammatory markers}

BMI was calculated by the following formula: weight $(\mathrm{kg}) /$ height $\left(\right.$ meters $\left.^{2}\right)$. Blood samples were collected from patients with schizophrenia between 06:00 and 08:00 AM after an overnight fast. The plasma was separated and then frozen at $-80^{\circ} \mathrm{C}$ until analysis. FBG levels were measured by glucose oxidase method (Meikang Biotech, Ningbo, China), high sensitivity CRP levels by immunoturbidimetric method (Leadman Biotech, Bejing, China), and IL- 6 and tumor necrosis factor-alpha (TNF-a) levels by cytometric bead array (CBA) method (BD Biosciences, San Diego, USA) in the clinical laboratory in Chaohu Hospital of Anhui Medical University. The detection range for CRP was 0.06-16.0 mg/dL, with inter- and intra-assay variation coefficients of $8 \%$ and $6 \%$, respectively. And the detection limits for IL- 6 and TNF-a were 1.6 and $0.7 \mathrm{pg} / \mathrm{mL}$, respectively.

\section{Data analysis}

Statistical analyses were conducted using SPSS (version 23.0). Kolmogorov-Smirnov test was used to detect the normality of the distribution of variables. Demographic and clinical variables were compared between different groups (patients with and without insomnia symptoms) by using chi-square test, independent sample t-test, and Mann-Whitney U-test, as appropriate. Furthermore, binary logistic regression analysis was performed to examine the related factors of insomnia in patients with schizophrenia by taking insomnia as the dependent variable and potential confounding factors with Pvalue $<0.10$ in univariate analyses as independent variables. Pearson or Spearman coefficients were further performed to explore the association between ISI components and other variables. Stepwise multiple regression was then performed to identify which factors were attributed to ISI. The significance level of all analyses was set at $P<0.05$ (2- tailed).

\section{Results}

\section{Participant characteristics}

Of the 331 inpatients with chronic schizophrenia recruited from the three hospitals, 328 patients (including 196 men and 132 women) completed all questionnaires and were included in the analysis. Table 1 shows that the average age was $45.1 \pm 11.8$ years (range, $19-74$ years) and the average education level was $8.1 \pm 3.6$ years (range, $0-18$ years). The proportion of patients taking a single firstgeneration (FG), second-generation (SG), and mixed antipsychotics was $2.1 \%(7 / 328), 41.7 \%(136 / 328)$, 
and $56.4 \%$ (185/328), respectively. In addition, $16.5 \%$ (54/328) of patients had at least one type of CSD, including 39 cases of diabetes and 28 cases of hypertension. 
Table 1

Socio-demographic and clinical characteristics of patients

\begin{tabular}{|c|c|c|c|c|c|c|c|c|c|}
\hline & \multicolumn{2}{|c|}{$\begin{array}{l}\text { Whole sample } \\
(n=328)\end{array}$} & \multicolumn{2}{|c|}{$\begin{array}{l}\text { Insomnia } \\
(n=126)\end{array}$} & \multicolumn{2}{|c|}{$\begin{array}{l}\text { Non-insomnia } \\
(n=202)\end{array}$} & \multicolumn{2}{|c|}{ Statistics } & \multirow[b]{2}{*}{$\mathrm{P}$} \\
\hline & N & $\%$ & $\mathrm{~N}$ & $\%$ & $\mathrm{~N}$ & $\%$ & $x^{2}$ & df & \\
\hline Male & 196 & 59.8 & 72 & 57.1 & 124 & 61.4 & 0.58 & 1 & 0.45 \\
\hline Married & 96 & 29.3 & 38 & 30.2 & 58 & 28.7 & 0.08 & 1 & 0.78 \\
\hline Smoking behavior & 98 & 29.9 & 31 & 24.6 & 67 & 33.2 & 2.72 & 1 & 0.10 \\
\hline Single FGA & 7 & 2.1 & 3 & 2.4 & 4 & 2.0 & 0 & 1 & 1.00 \\
\hline Single SGA & 136 & 41.7 & 49 & 38.9 & 87 & 43.1 & 0.56 & 1 & 0.46 \\
\hline BZDs & 62 & 18.9 & 28 & 22.2 & 34 & 16.8 & 1.47 & 1 & 0.23 \\
\hline CSDs & 54 & 16.5 & 24 & 19.0 & 30 & 14.9 & & & \\
\hline Diabetes & 39 & 11.9 & 15 & 11.9 & 24 & 11.9 & 0 & 1 & 1.00 \\
\hline \multirow[t]{2}{*}{ Hypertension } & 28 & 8.5 & 16 & 12.7 & 12 & 5.9 & 4.54 & 1 & 0.03 \\
\hline & Mean & SD & Mean & SD & Mean & SD & $\mathrm{T} / \mathrm{Z}$ & $\mathrm{df}$ & $P$ \\
\hline Age (years) & 45.1 & 11.8 & 47.0 & 11.7 & 43.9 & 11.7 & 2.40 & 326 & 0.02 \\
\hline Education (years) & 8.1 & 3.6 & 8.0 & 3.4 & 8.2 & 3.8 & $-0.24^{a}$ & & 0.81 \\
\hline Age of onset (years) & 26.0 & 8.2 & 26.3 & 7.9 & 25.7 & 8.4 & 0.66 & 326 & 0.51 \\
\hline $\begin{array}{l}\text { Duration of illness } \\
\text { (years) }\end{array}$ & 19.1 & 10.4 & 20.6 & 11.0 & 18.1 & 10.0 & $-1.97^{a}$ & & 0.049 \\
\hline $\begin{array}{l}\text { Number of } \\
\text { hospitalizations }\end{array}$ & 5.7 & 4.9 & 5.7 & 5.5 & 5.7 & 4.6 & $-0.32^{a}$ & & 0.75 \\
\hline CPZeq (mg/day) & 455.3 & 261.7 & 453.6 & 237.8 & 455.0 & 275.6 & $-0.65^{a}$ & & 0.51 \\
\hline $\begin{array}{l}\text { Dose stabilization } \\
\text { (weeks) }\end{array}$ & 11.7 & 10.4 & 11.0 & 9.1 & 12.0 & 11.1 & $-0.60^{a}$ & & 0.55 \\
\hline $\mathrm{BMI}\left(\mathrm{kg} / \mathrm{m}^{2}\right)$ & 24.1 & 3.8 & 24.4 & 3.8 & 23.9 & 3.9 & 1.16 & 326 & 0.25 \\
\hline
\end{tabular}

a Mann-Whitney U-test; Statistical differences are bold.

FGA, first-generation antipsychotic; SGA, second-generation antipsychotic; CSD, chronic somatic diseasese; BZD, benzodiazepine; CPZeq, chlorpromazine equivalent; FBG, fasting blood glucose; BMI, Body Mass Index; PANSS-P, Positive subscore of PANSS; PANSS-N, Negative subscore of PANSS; PANSS-G, General psychopathology subscore of PANSS; CDSS, Calgary Depression Scale for Schizophrenics, minus the sleep item. 


\begin{tabular}{|c|c|c|c|c|c|c|c|c|c|}
\hline \multirow[b]{2}{*}{ FBG (mmol/L) } & \multicolumn{2}{|c|}{$\begin{array}{l}\text { Whole sample } \\
(n=328)\end{array}$} & \multicolumn{2}{|c|}{$\begin{array}{l}\text { Insomnia } \\
(n=126)\end{array}$} & \multicolumn{2}{|c|}{$\begin{array}{l}\text { Non-insomnia } \\
(n=202)\end{array}$} & \multicolumn{3}{|c|}{ Statistics } \\
\hline & 5.3 & 1.4 & 5.6 & 1.6 & 5.2 & 1.2 & $-2.47^{a}$ & & 0.01 \\
\hline PANSS-P & 17.9 & 7.3 & 19.1 & 6.9 & 17.1 & 7.5 & $-2.66^{a}$ & & 0.008 \\
\hline PANSS-N & 21.6 & 7.6 & 21.7 & 6.6 & 21.5 & 8.2 & 0.33 & 326 & 0.74 \\
\hline PANSS-G & 38.3 & 12.7 & 40.6 & 11.6 & 36.9 & 13.1 & 2.65 & 326 & 0.009 \\
\hline CDSS & 3.0 & 3.1 & 4.2 & 3.3 & 2.3 & 2.8 & $\begin{array}{l}-6.43 \\
a\end{array}$ & & $\dot{\delta} 001$ \\
\hline \multicolumn{10}{|c|}{ a Mann-Whitney U-test; Statistical differences are bold. } \\
\hline \multicolumn{10}{|c|}{$\begin{array}{l}\text { FGA, first-generation antipsychotic; SGA, second-generation antipsychotic; CSD, chronic somatic } \\
\text { diseasese; BZD, benzodiazepine; CPZeq, chlorpromazine equivalent; FBG, fasting blood glucose; BMI, } \\
\text { Body Mass Index; PANSS-P, Positive subscore of PANSS; PANSS-N, Negative subscore of PANSS; } \\
\text { PANSS-G, General psychopathology subscore of PANSS; CDSS, Calgary Depression Scale for } \\
\text { Schizophrenics, minus the sleep item. }\end{array}$} \\
\hline
\end{tabular}

\section{Prevalence of insomnia symptoms}

The prevalence rate of $\geq 1$ type of insomnia symptoms among all patients was $38.4 \%$ (126/328), and the prevalence of early, middle and late insomnia was $26.2 \%$ (86/328), $21.6 \%(71 / 328)$, and $17.1 \%(56 / 328)$, respectively. Although the prevalence of insomnia symptoms in females appeared to be higher than that in males $(40.9 \%$ versus $36.7 \%)$, the difference was not statistically significant $\left(\chi^{2}=0.58, \mathrm{df}=1, P=0.45\right)$.

\section{Demographic and clinical variables between insomnia and non-insomnia groups}

Compared with those without insomnia symptoms, patients with insomnia symptoms had older age, longer duration of illness, higher proportion of hypertension, higher levels of FBG, as well as more positive (PANSS-P), general psychopathology (PANSS-G), and depressive symptoms (CDSS) (Table 1). Further, binary logistic regression analysis showed that only depressive symptoms (CDSS) were still associated with insomnia symptoms $(\mathrm{OR}=1.23,95 \% \mathrm{Cl}: 1.13-1.33, P<0.001)$. In addition, there were no significant differences in CRP, IL-6, or TNF-a levels between the insomnia and non-insomnia groups (Table 2). 
Table 2

Inflammatory markers

\begin{tabular}{|lllll|}
\hline Inflammatory markers & $\begin{array}{l}\text { Insomnia } \\
(\mathbf{n = 1 2 6})\end{array}$ & $\begin{array}{l}\text { Non-insomnia } \\
(\mathbf{n}=\mathbf{2 0 2})\end{array}$ & $\mathrm{Z}^{\mathrm{a}}$ & P value \\
\hline $\mathrm{CRP}(\mathrm{mg} / \mathrm{L})$ & $2.46 \pm 4.00^{\mathrm{b}}$ & $2.46 \pm 8.02^{\mathrm{c}}$ & -0.85 & 0.40 \\
\hline $\mathrm{IL}-6(\mathrm{pg} / \mathrm{ml})$ & $1.65 \pm 1.59^{\mathrm{c}}$ & $1.68 \pm 2.14^{\mathrm{d}}$ & -0.89 & 0.38 \\
\hline TNF-a $(\mathrm{pg} / \mathrm{ml})$ & $0.39 \pm 0.33^{\mathrm{c}}$ & $0.48 \pm 0.86^{\mathrm{d}}$ & -1.08 & 0.28 \\
\hline${ }^{a}$ Mann-Whitney U-test; Missing information for $^{\mathrm{b}} 2{ }^{2}{ }^{\mathrm{c}} 3$, and $^{\mathrm{d}} 5$ patients. \\
\hline
\end{tabular}

\section{Associations between ISI and demographic and clinical variables}

Compared with patients without insomnia symptoms, patients with insomnia symptoms had higher ISI score ( $7.5 \pm 4.6$ versus $1.4 \pm 1.4 ; Z=-13.55, P<0.001)$. There was no significant difference in ISI score between male and female, married and non-married, or smoking and non-smoking patients (all $P>0.05$ ).

Correlation analysis showed that ISI score was significantly associated with age $(r=0.13, P<0.05)$, blood glucose level $(r=0.14, P<0.05)$, PNASS positive symptoms $(r=0.19, P<0.001)$, general psychopathology $(r=0.17, P<0.01$, PANSS total score $(r=0.18, P<0.01)$, and depressive symptom shown on CDSS $(r=$ $0.43, P<0.001$ ) (Table 3). Further stepwise multiple regression showed that higher CDSS score (beta $=$ $0.55, \mathrm{t}=8.21, P<0.001$ ) and older age (beta $=0.06, \mathrm{t}=3.59, P<0.001$ ) were significantly associated with higher ISI score, while taking a single SGA (beta $=-0.85, t=-1.99, P<0.05$ ) was associated with a lower ISI score (Table 4). 
Table 3

Correlations between the components of ISI and demographic and clinical measures

\begin{tabular}{|c|c|c|c|c|c|c|c|c|}
\hline & Age & Hypertension & $\begin{array}{l}\text { Duration } \\
\text { of } \\
\text { illness }\end{array}$ & FBG & $\begin{array}{l}\text { PANSS- } \\
\mathrm{P}\end{array}$ & $\begin{array}{l}\text { PANSS- } \\
\mathbf{N}\end{array}$ & $\begin{array}{l}\text { PANSS- } \\
\text { G }\end{array}$ & CDSS \\
\hline ISI-1 & $0.11^{*}$ & $0.11^{*}$ & 0.09 & 0.09 & $0.25^{\star \star \star}$ & $0.13^{*}$ & $0.24^{\star \star \star}$ & $0.40^{\star \star \star}$ \\
\hline ISI-2 & $0.16^{\star \star}$ & $0.13^{*}$ & 0.10 & $0.16^{\star \star}$ & $0.14^{\star \star}$ & 0.07 & $0.14^{*}$ & $0.37^{\star \star \star}$ \\
\hline ISI-3 & $0.13^{*}$ & 0.09 & $0.12^{*}$ & 0.10 & $0.18^{\star *}$ & $0.13^{*}$ & $0.18^{\star *}$ & $0.43^{\star * \star}$ \\
\hline $\begin{array}{l}\text { ISI } \\
\text { Total }\end{array}$ & $0.13^{*}$ & 0.10 & 0.10 & $0.14^{\star}$ & $0.19^{\star \star \star}$ & 0.11 & $0.17^{* \star}$ & $0.43^{\star \star \star}$ \\
\hline \multicolumn{9}{|c|}{$\begin{array}{l}\text { FBG, fasting blood glucose; ISI-1, Factor } 1 \text { labeled as "Impact" in ISI; ISI-2, Factor } 2 \text { labeled as } \\
\text { "Severity" in ISI; Factor } 3 \text { labeled as "Satisfaction" in ISI; PANSS-P, Positive subscore of PANSS; } \\
\text { PANSS-N, Negative subscore of PANSS; PANSS-G, General psychopathology subscore of PANSS; } \\
\text { CDSS, Calgary Depression Scale for Schizophrenics, minus the sleep item. }\end{array}$} \\
\hline \multicolumn{9}{|c|}{${ }^{*} P<0.05}$. \\
\hline \multicolumn{9}{|c|}{${ }^{* *} P<0.01}$. \\
\hline ** & & & & & & & & \\
\hline
\end{tabular}

Table 4

Factors associated ISI score by Stepwise Multiple Regression

\begin{tabular}{|lllllll|}
\hline & \multicolumn{3}{l}{ Coefficients } & T & P & \multicolumn{2}{l|}{ 95\% Cl for B } \\
\cline { 2 - 3 } \cline { 6 - 7 } & B & Std. Error & & & Lower Bound & Upper Bound \\
\cline { 1 - 2 } (Constant) & -0.46 & 0.88 & -0.52 & 0.60 & -2.19 & 1.27 \\
\hline CDSS & 0.55 & 0.07 & 8.21 & $<0.001$ & 0.42 & 0.68 \\
\hline Age & 0.06 & 0.02 & 3.59 & $<0.001$ & 0.03 & 0.10 \\
\hline Single SGA & -0.85 & 0.43 & -1.99 & 0.048 & -1.69 & -0.01 \\
\hline Cl, confidence interval; SGA, second-generation antipsychotic. & \\
\hline
\end{tabular}

In addition, correlation analysis showed no significant correlation between ISI score and any of inflammatory markers (CRP, IL-6, or TNF-a; all $P>0.05$ ) (Table 5). 
Table 5

ISI and Inflammatory markers

\begin{tabular}{|llll|}
\hline & CRP & IL-6 & TNF-a \\
\hline ISI-1 & $0.04(0.51)$ & $-0.16(0.77)$ & $0.03(0.63)$ \\
\hline ISI-2 & $0.04(0.46)$ & $0.01(0.81)$ & $-0.08(0.18)$ \\
\hline ISI-3 & $0.02(0.74)$ & $0.00(0.96)$ & $-0.02(0.78)$ \\
\hline ISI Total & $0.03(0.60)$ & $0.00(0.95)$ & $-0.02(0.73)$ \\
\hline Data are presented as correlation coefficient and P value. \\
\hline
\end{tabular}

\section{Discussion}

To the best of our knowledge, this was the first study to investigate the prevalence and clinical correlates of insomnia symptoms, and to explore the relationship between insomnia and inflammatory markers in patients with chronic schizophrenia in China. There were several main fingings of this study: 1) we found a high rate of insomnia symptoms in patients with chronic schizophrenia; 2) compared with patients without insomnia symptoms, patients with insomnia symptoms had older age, longer duration of illness, higher proportion of hypertension, higher levels of FBG, more psychotic and depressive symptoms; 3 ) some demographic and clinical variables were found to be risk factors for insomnia in these patients, including more severe depressive symptoms and older age, while taking a single SGA was a beneficial factor; 4) there was no significant correlation between insomnia symptoms and any inflammatory markers.

The prevalence of insomnia symptoms in hospitalized patients with chronic schizophrenia was $38.4 \%$, which was close to the results of previous studies in Chinese outpatients $(36.0 \%)$ and community patients $(28.9 \%)$ with schizophrenia $[9,18]$, but lower than that in a study of 175 outpatients with schizophrenia or schizophrenic affective disorder from the USA (44\%), adopting more stringent criteria for insomnia with $\mid S I \geq 15$ [28]. This difference can be considered as a considerable regional difference in the rate and severity off insomnia between Chinese and Western populations [30]. Also, this can be explained by their different hours of work and rest, or their different perception of insomnia, such as sensitivity to insomnia.

Furthermore, the prevalence of early, middle and late insomnia symptoms found in this study were very close to the corresponding $21.1 \%, 23.6 \%$ and $11.9 \%$, as well as $20.5 \%, 19.6 \%$ and $17.7 \%$ in the previous two studies $[9,18]$. These results confirmed that difficulty falling asleep and maintaining sleep are the two most common manifestations of insomnia in schizophrenia [11]. In a Chinese epidemiologic survey [31] using insomnia criteria similar to this study, the prevalence rates of early, middle, late, and any type of insomnia symptoms in general population were $7.0 \%, 8.0 \%, 4.9 \%$ and $9.2 \%$, respectively. Therefore, the prevalence of insomnia symptoms in inpatients with schizophrenia is higher than that in normal people. 
As a result, hospitalized patients with schizophrenia are prone to insomnia and require more attention, even if they receive regular medical rounds and care.

Our current study found that schizophrenia patients with insomnia symptoms had significantly older age and longer duration of illness than those without insomnia symptoms. Further correlation analysis and showed that the ISI score was positively associated with the patients' age, which was confirmed by stepwise multiple regression. These results confirmed the findings of previous studies $[9,18]$. In addition, we found that ISI-3 (Satisfaction) was positively associated with duration of illness, indicating that patients with longer duration of illness had lower satisfaction with sleep in schizophrenia patients. Taken together, these findings suggest that older age and longer duration illness may be risk factors for sleep disturbances in patients with chronic schizophrenia.

Further, we found that FBG level was significantly higher in the insomnia group than that in the noninsomnia group. Moreover, FGB level was positively correlated with ISI score and ISI-2 (Severity). Our results suggest that schizophrenia patients with higher FBG levels were more likely to suffer from insomnia than those with normal FBG levels. In a large epidemiological study, about $25 \%$ patients with type 2 diabetes were diagnosed with sleep disorders, and more than 75\% reported sleep symptoms [32]. A number of observational studies have shown an association between metabolic disturbance and sleep disorders [33]. These results suggest that abnormal glucose metabolism or diabetes may have a significant impact on sleep in both schizophrenia patients and general population.

In addition, we found that the proportion of hypertension in patients with insomnia symptoms was higher than that in patients without insomnia symptoms, indicating that insomnia is associated with hypertension in schizophrenia. A previous study confirmed that patients with chronic insomnia had an increased cardiovascular risk compared with healthy controls [34]. This may be related to the dysregulation of hypothalamic-pituitary axis, increased activity of sympathetic nervous system, and elevated inflammatory level [35].

Our results showed that patients with insomnia symptoms had more severe positive and general psychopathological symptoms than those without insomnia symptoms, which was consistent with the results of several previous studies. For example, a previous study found that sleep disturbances predicted the greater auditory hallucinations, paranoia and delusions the next day in schizophrenia [36], suggesting that insomnia or poor sleep quality is associated with worse psychotic symptoms in patients with schizophrenia. Moreover, several studies have found that insomnia was associated with positive $[9,18]$, negative [18], anxiety $[9,18]$ and depressive symptoms $[9,18,28]$ in patients with schizophrenia. Among the schizophrenia patients in this study, the correlation remained significant after removal of the overlapping item with insomnia in CDSS. More severe depressive symptoms were independently associated with a higher risk of insomnia and higher ISI scores, suggesting that they are risk factors for insomnia. Additionally, the multiple regression showed that taking a single SGA was associated with a lower ISI score, which may be a protective factor for insomnia in the patients with schizophrenia. 
Of note, we found no significant correlation between inflammatory markers and insomnia or ISI score in patients with chronic schizophrenia. In contrast, two previous studies found higher levels of inflammatory measures (neutrophil-lymphocyte and platelet-lymphocyte ratios) [16] and higher levels of CRP and IL-6 [17] in schizophrenia patients with poor sleep quality. The first study assessed inflammation and sleep quality differently from this study. And the participants in the latter study were outpatients with schizophrenia, whose lifestyles and sleep habits may differ from those of inpatients with chronic schizophrenia. Moreover, although the relationship between schizophrenia and inflammatory abnormalities has been repeatedly confirmed [37], the levels of inflammatory cytokines are easily affected by a variety of confounding factors, such as gender, stress, course of disease and the administration of different antipsychotics [37-39]. Therefore, the relationship between sleep disturbances and inflammation in patients with schizophrenia needs to be further studied.

Several methodological limitations of this study should be noted. First, this was a cross-sectional design that did not identify the direction and causal relationship between demographic or clinical variables and insomnia or ISI score in patients with schizophrenia. Second, all participants were recruited from three hospitals in the same province, which led to in a lack of nationwide representation. Third, insomnia was defined by loose criteria rather than an objective measure of sleep, such as recording through polysomnography or actigraphy. Finally, there was no healthy control group in this study, and we were unable to make a direct comparison between schizophrenia and normal populations.

\section{Conclusions}

In conclusion, insomnia is common in hospitalized patients with schizophrenia in China, especially those with older age, or more depressive symptoms. Taking a single SGA may be a protective factor for insomnia. In addition, there is no clear correlation between insomnia symptoms and inflammation in schizophrenia. Due to methodological limitations, including a small sample size, chronic inpatients, no matched healthy controls, and no objective measurements for sleep, our results should be considered preliminary. Longitudinal studies with more representative samples will help to further clarify the relationship of insomnia with its risk factors and inflammation in patients with schizophrenia, and provide reasonable prevention and intervention measures for clinical practice.

\section{Abbreviations}

AP: antipsychotic; BMI: Body Mass Index; BZD: benzodiazepine; CBA: cytometric bead array; CDSS: Calgary Depression Scale for Schizophrenia; CPZeq, chlorpromazine equivalent; CSDs: chronic somatic diseases; DDDs, defined daily doses; DSM-IV: 4th edition of Diagnostic and Statistical Manual of Mental Disorder; FBG: fasting blood glucose; FGA: first-generation antipsychotic; HAM-D: Hamilton Depression Rating Scale; ICD-10: 10th revision of the International Statistical Classification of Diseases and Related Health Problems; ISI: Insomnia Severity Index; PANSS: Positive and Negative Syndrome Scale; SGA: second-generation antipsychotic; TNF-a: factor-alpha. 


\section{Declarations}

\section{Acknowledgements}

The authors would like to thank Dr. Mei Zhu for assistance in the performance of the measurements of fasting blood glucose and inflammatory cytokines levels.

\section{Authors' contributions}

Study design and funding acquisition: HL. Data collection, analysis and interpretation: $L X, Y Z, Z L, Y Z, K Z$. Drafting of the manuscript: LX, YZ. Critical revision of the manuscript: XYZ. Approval of the final version for publication: all co-authors.

\section{Funding}

The study was supported by the Anhui Province Key Scientific and Technological Projects (1804h08020263, 202004j07020030), the Scientific Research Fundation of the Institute for Translational Medicine (2017zhyx17), the National Clinical Key Specialty Project Foundation (CN), the National Natural Science Foundation of China (81801341).

\section{Availability of data and materials}

The datasets used and analysed for this study are available from the corresponding author on reasonable request.

\section{Ethics approval and consent to participate}

The Ethics Committee of Chaohu Hospital of Anhui Medical University approved the protocol before the study began. All participants in this study provided written informed consent.

\section{Consent for publication}

All participants provided written informed consent for publication.

\section{Competing interests}

The authors declare that they have no conflict of interest. 


\section{References}

1. Buysse DJ. Insomnia. JAMA 2013;309:706-716.

2. Ohayon MM. Epidemiology of insomnia: what we know and what we still need to learn. Sleep Med Rev 2002;6:97-111.

3. Morin CM, LeBlanc M, Daley M, Gregoire JP, Merette C. Epidemiology of insomnia: prevalence, selfhelp treatments, consultations, and determinants of help-seeking behaviors. Sleep Med 2006;7:123130.

4. Uhlig BL, Sand T, Odegard SS, Hagen K. Prevalence and associated factors of DSM-V insomnia in Norway: the Nord-Trondelag Health Study (HUNT 3). Sleep Med 2014;15:708-713.

5. Leger D, Bayon V. Societal costs of insomnia. Sleep Med Rev 2010;14:379-389.

6. Hombali A, Seow E, Yuan Q, Chang SHS, Satghare P, Kumar S, et al. Prevalence and correlates of sleep disorder symptoms in psychiatric disorders. Psychiatry Res 2018.

7. Kaskie RE, Graziano B, Ferrarelli F. Schizophrenia and sleep disorders: links, risks, and management challenges. Nat Sci Sleep 2017;9:227-239.

8. Lunsford-Avery JR, Goncalves B, Brietzke E, Bressan RA, Gadelha A, Auerbach RP, et al. Adolescents at clinical-high risk for psychosis: Circadian rhythm disturbances predict worsened prognosis at 1year follow-up. Schizophr Res 2017;189:37-42.

9. Xiang YT, Weng YZ, Leung CM, Tang WK, Lai KY, Ungvari GS. Prevalence and correlates of insomnia and its impact on quality of life in Chinese schizophrenia patients. Sleep 2009;32:105-109.

10. Miller BJ, Parker CB, Rapaport MH, Buckley PF, McCall WV. Insomnia and suicidal ideation in nonaffective psychosis. Sleep 2019;42.

11. Monti JM, BaHammam AS, Pandi-Perumal SR, Bromundt V, Spence DW, Cardinali DP, et al. Sleep and circadian rhythm dysregulation in schizophrenia. Prog Neuropsychopharmacol Biol Psychiatry 2013;43:209-216.

12. Monti JM, Torterolo P, Pandi Perumal SR. The effects of second generation antipsychotic drugs on sleep variables in healthy subjects and patients with schizophrenia. Sleep Med Rev 2017;33:51-57.

13. Irwin MR, Opp MR. Sleep Health: Reciprocal Regulation of Sleep and Innate Immunity. Neuropsychopharmacology 2017;42:129-155.

14. Irwin MR, Olmstead R, Carroll JE. Sleep Disturbance, Sleep Duration, and Inflammation: A Systematic Review and Meta-Analysis of Cohort Studies and Experimental Sleep Deprivation. Biol Psychiatry 2016;80:40-52.

15. Krysta K, Krzystanek M, Bratek A, Krupka-Matuszczyk I. Sleep and inflammatory markers in different psychiatric disorders. J Neural Transm (Vienna) 2017;124:179-186.

16. Fang SH, Suzuki K, Lim CL, Chung MS, Ku PW, Chen LJ. Associations between sleep quality and inflammatory markers in patients with schizophrenia. Psychiatry Res 2016;246:154-160. 
17. Lee EE, Ancoli-Israel S, Eyler LT, Tu XM, Palmer BW, Irwin MR, et al. Sleep Disturbances and Inflammatory Biomarkers in Schizophrenia: Focus on Sex Differences. Am J Geriatr Psychiatry 2019;27:21-31.

18. Hou CL, Li Y, Cai MY, Ma XR, Zang Y, Jia FJ, et al. Prevalence of Insomnia and Clinical and Quality of Life Correlates in Chinese Patients With Schizophrenia Treated in Primary Care. Perspect Psychiatr Care 2017;53:80-86.

19. Li SX, Lam SP, Zhang J, Yu MW, Chan JW, Chan CS, et al. Sleep Disturbances and Suicide Risk in an 8-Year Longitudinal Study of Schizophrenia-Spectrum Disorders. Sleep 2016;39:1275-1282.

20. WHO. The ICD-10 Classification of Mental and Behavioral Disorders: Clinical Descriptions and Diagnostic Guidelines. Geneva, 1992.

21. Leucht S, Samara M, Heres S, Davis JM. Dose Equivalents for Antipsychotic Drugs: The DDD Method. Schizophr Bull 2016;42 Suppl 1:S90-94.

22. Xiang YT, Wong TS, Tsoh J, Ungvari GS, Correll CU, Ko FW, et al. Insomnia in older adults with chronic obstructive pulmonary disease (COPD) in Hong Kong: a case-control study. COPD 2014;11:319-324.

23. Olie JP, Kasper S. Efficacy of agomelatine, a MT1/MT2 receptor agonist with 5-HT2C antagonistic properties, in major depressive disorder. Int J Neuropsychopharmacol 2007;10:661-673.

24. Bastien $\mathrm{CH}$, Vallieres $\mathrm{A}$, Morin $\mathrm{CM}$. Validation of the Insomnia Severity Index as an outcome measure for insomnia research. Sleep Med 2001;2:297-307.

25. Morin CM, Belleville G, Belanger L, Ivers $H$. The Insomnia Severity Index: psychometric indicators to detect insomnia cases and evaluate treatment response. Sleep 2011;34:601-608.

26. Chen PY, Yang CM, Morin CM. Validating the cross-cultural factor structure and invariance property of the Insomnia Severity Index: evidence based on ordinal EFA and CFA. Sleep Med 2015;16:598-603.

27. Xiao W, Liu H, Zhang H, Liu Q, Fu P, Chen J, et al. Reliability and validity of the Chinese version of the Calgary Depression Scale for Schizophrenia. Aust N Z J Psychiatry 2009;43:548-553.

28. Palmese LB, DeGeorge PC, Ratliff JC, Srihari VH, Wexler BE, Krystal AD, et al. Insomnia is frequent in schizophrenia and associated with night eating and obesity. Schizophr Res 2011;133:238-243.

29. Kay SR, Fiszbein A, Opler LA. The positive and negative syndrome scale (PANSS) for schizophrenia. Schizophr Bull 1987;13:261-276.

30. Cao XL, Wang SB, Zhong BL, Zhang L, Ungvari GS, Ng CH, et al. The prevalence of insomnia in the general population in China: A meta-analysis. PLoS One 2017;12:e0170772.

31. Xiang YT, Ma X, Cai ZJ, Li SR, Xiang YQ, Guo HL, et al. The prevalence of insomnia, its sociodemographic and clinical correlates, and treatment in rural and urban regions of Beijing, China: a general population-based survey. Sleep 2008;31:1655-1662.

32. Gupta S, Wang Z. Predictors of sleep disorders among patients with type 2 diabetes mellitus. Diabetes Metab Syndr 2016;10:213-220.

33. Schmid SM, Hallschmid M, Schultes B. The metabolic burden of sleep loss. Lancet Diabetes Endocrinol 2015;3:52-62. 
34. Carter JR, Grimaldi D, Fonkoue IT, Medalie L, Mokhlesi B, Cauter EV. Assessment of sympathetic neural activity in chronic insomnia: evidence for elevated cardiovascular risk. Sleep 2018;41.

35. Javaheri S, Redline S. Insomnia and Risk of Cardiovascular Disease. Chest 2017;152:435-444.

36. Mulligan LD, Haddock G, Emsley R, Neil ST, Kyle SD. High resolution examination of the role of sleep disturbance in predicting functioning and psychotic symptoms in schizophrenia: A novel experience sampling study. J Abnorm Psychol 2016;125:788-797.

37. Kirkpatrick B, Miller BJ. Inflammation and schizophrenia. Schizophr Bull 2013;39:1174-1179.

38. O'Connell KE, Thakore J, Dev KK. Pro-inflammatory cytokine levels are raised in female schizophrenia patients treated with clozapine. Schizophr Res 2014;156:1-8.

39. Stapel B, Sieve I, Falk CS, Bleich S, Hilfiker-Kleiner D, Kahl KG. Second generation atypical antipsychotics olanzapine and aripiprazole reduce expression and secretion of inflammatory cytokines in human immune cells. J Psychiatr Res 2018;105:95-102. 